\title{
The orbit design and control strategy model of Chang'e-3 in the main reduction process
}

\author{
Rui-fang Ji \\ School of North China Electric Power University, Baoding 071000, China; \\ 598561035@qq.com
}

Keywords: uniform three-dimensional sphere model, optimal trajectory, main reduction section

\begin{abstract}
On December 14, 2013, Chang'e-3 set down on the moon, making China the third country in the world to set a soft landing with a lunar probe. It is a great honor for us Chinese, but also a great challenge for the project staff through the whole process. Of the characteristics of automatic navigation control, the moon landing stage is considered to be the most important test. In this paper, the dynamic model based on the uniform sphere three-dimensional soft landing model is established to searching Chang'e-3's optimal trajectory in the main reduction process along the moon landing stage. Also, the computer simulation method and the exhaustive method are employed. Compared with the searched video information, the accuracy of the results is verified.
\end{abstract}

\section{Introduction}

Chang'e-3 launched on December 12, 2013 and successfully landed on the moon two days later. It is the first time for china to send a spacecraft land on the surface of an extraterrestrial body and conduct surveys on the moon. Furthermore, the success makes China the third country to carry out such a rover mission after the United States and former Soviet Union.

For there is no air on the moon, parachute is unavailable. Chang'e-3 has to rely on a variable thrust engine, to complete the soft landing task in the mid-course corrections, the braking at perilune, the powered lowering, and the hovering period. At this stage, the measurement and control technology can not keep up with the operation of the satellite. As a consequence, Chang'e-3, armed with the program set in advance, depend entirely on autonomous navigation control, reducing the height, determining the landing site, achieving the soft landing and a series of key actions.

In this paper, the dynamic model based on the uniform sphere three-dimensional soft landing model is established to solve Chang'e-3's optimal trajectory in the main reduction section. The optimization goal is the fuel consumption, and is transferred to the seeking for the shortest time, for the largest thrust remains the same in the main reduction stage. Then, the exhaustive method list three kinds of movements: constant horizontal thrust, constant speed reverse thrust and constant speed reverse thrust after constant speed reverse resultant force., and only to find that just the last one state meet the key position after simulation. Then, the computer simulation method is employed, and the movement of the main reduction process is given. Compared with the searched video information, the accuracy of the results is verified.

The dynamic model based on the uniform sphere three-dimensional soft landing model [2]

- Symbols and definitions

\author{
Symbols Definitions
}




\begin{tabular}{ll}
\hline$a_{\max }$ & the largest horizontal reverse acceleration \\
$t_{\min }$ & the shortest time that the horizontal speed reduce to 0 \\
$h_{\min }$ & the land's vertical landing altitude in the process \\
$\mu \mathrm{m}$ & the moon's gravitational constant \\
$I$ & the specific impulse of propulsion system \\
$G$ & the acceleration of gravity of the earth's surface \\
$a_{F}$ & brake thrust acceleration \\
$a_{m}$ & the moon's gravitational acceleration \\
$\phi$ & the main reduction period of latitude deflection angle
\end{tabular}

\section{- Model building}

1) Establish the coordinate system

The moon heart inertial reference coordinate system OXYZ: the origin of the coordinate system 0 is located in the heart. The $\mathrm{Z}$ axis directs from the moon heart to the initial landing point, while the $\mathrm{X}$ axis in lunar orbit plane points in the heading direction. The $\mathrm{Y}$ axis constitutes the rectangular coordinate system with the and $\mathrm{X}$ axis and $\mathrm{Z}$ axis, which is shown in figure 1 .

Descending track reference coordinate system oxyz: origin 0 is located in the lander centroid, and the $y$ axis directs from the moon heart to lander centroid, while the $\mathrm{x}$ axis in the local water points to the lander's heading direction. The $\mathrm{z}$ axis constitutes the rectangular coordinate system with the $\mathrm{x}$ axis and y axis. The two thrust Angle between the constant thrust F and xyz coordinate system oxyz are thrust bearing $\varphi$ and thrust angle $\theta$ respectively. It is the positive while the thrust bearing rotates around the positive $\mathrm{x}$ axis and the thrust angle rotates around the negative $\mathrm{x}$ axis. As shown in figure 2.

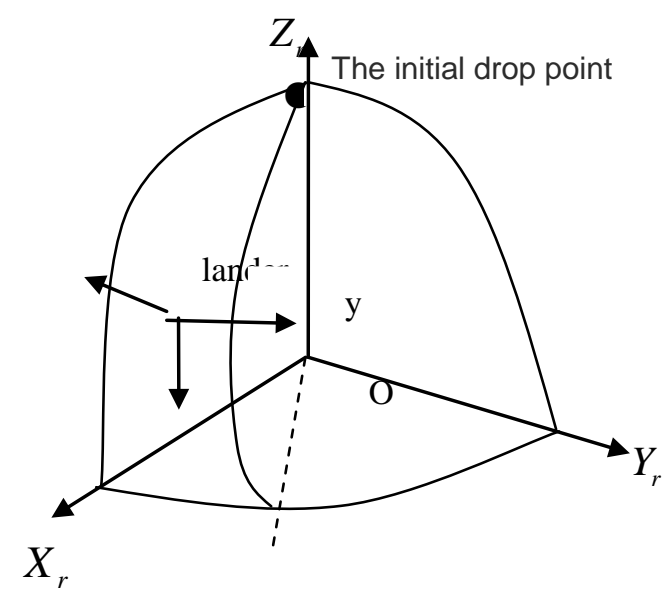

Fig1.The moon heart inertial reference coordinate system

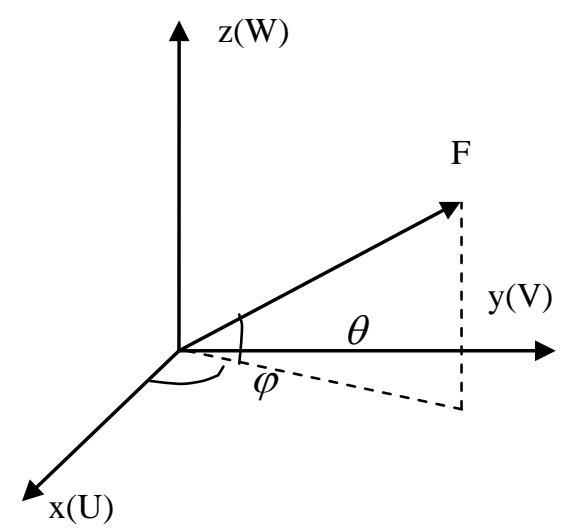


2) Building model

Fig2. Descending track reference coordinate system

$\mathrm{U}, \mathrm{V}, \mathrm{W}$ represent the lander's descent velocity component in the oxoyozo three axis respectively. Ignoring the moon's rotation and introducing quality equation at the same time, soft landing dynamic model can obtained employing the relationship between the ball coordinate system and the rectangular coordinates.it is shown as below:

$$
\begin{gathered}
\dot{U}=F \cos \theta \cos \varphi / m-U W / r+V^{2} /(r \tan \beta) \quad \dot{V}=F \cos \theta \sin \varphi / m-V W / r-U V /(r \tan \beta) \\
\dot{W}=F \sin \theta / m-\mu_{m} / r^{2}+\left(U^{2}+V^{2}\right) / r \\
\dot{r}=W, \dot{\alpha}=V /(r \sin \beta), \dot{\beta}=U / r, \dot{m}=-F /(I g)
\end{gathered}
$$

Equations (1) is the dynamic model of a soft landing throughout the entire flight.To equations (1), the nonlinear dynamic model, it is usually by iterating a given initial to work out the association state variables or the intermediate variables, and finally obtain the optimal control $\tilde{u}=(\theta, \varphi)^{T}$. This method goes against the realization of the independent control on the lander. Here, we base on the current state to make single step optimization to the thrust angle control quantity, that is to make local optimization in the rest of the time interval $\left[0,{ }_{s} t_{s}\right]$, and $\tilde{u}=(\theta, \varphi)^{T}$ is constantly updated through every step of the optimization calculation. Introducing the plane moon and the quality the same assumptions, equations (1), the dynamic model, can be simplified to

$$
\dot{x}=v, \dot{v}=a_{F}+a_{m}
$$

Where: $a_{F}=a_{F}(\cos \theta \cos \varphi, \cos \theta \sin \varphi, \sin \theta)^{T} \quad v=(U, V, W)^{T} \quad a_{m}=\left(0,0,-g_{m}\right)^{T}$

In the Braking period the fuel consumption is the optimal objective, which can be converted to the shortest braking time. That is to solve the control volume, which makes the lander transfer from the initial state to the terminal state, with the shortest time. Here, the initial value changes continuously with each step optimization. Terminal restrain including the speed and height constraints. Using the optimal control theory, the analytical expression of the two thrust Angle control quantity is given:

$$
\varphi=\tan ^{-1}\left(\left(V_{f b}-V\right) /\left(U_{f b}-U\right)\right) \quad \theta=\sin ^{-1}\left[\left(a_{r}+\mu_{m} / r^{2}-\left(U^{2}+V^{2}\right) / r\right) / a_{r}\right]
$$

where the subscript $\mathrm{f}$ indicates the terminal condition, and the subscript $\mathrm{b}$ represents the brake section, $\mathrm{r} 、 \mathrm{U} 、 \mathrm{~V}$ are decline parameters for the current moment; ${ }^{a_{r}}$ is the current radial acceleration.

Equations (3) shows the thrust angle guidance commands of the single step optimization. For that the moon's rotation angular velocity is small, and the main braking time is only a few hundred seconds, the changes in the longitude of the process is ignored. So in the above equations $\varphi$ equals 0 , and the three-dimensional dynamic process is reduced to two-dimensional space to analyze, which is shown as below:

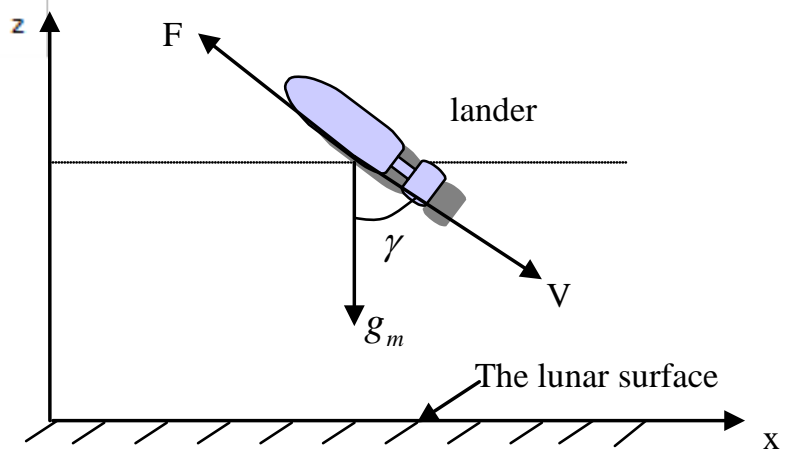

Fig3 .Two-dimensional space soft landing model figure 

Where $\gamma=\tan ^{-1} \frac{v_{z}}{v_{x}}, v_{z}$ denotes the speed component in the direction $z$, while $v_{x}$ indicates the

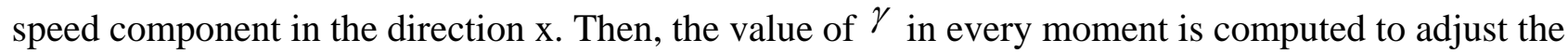 lander's thrust direction, through which the dynamic movement process of this phase is obtained.

\section{The test to three movements}

\section{- Constant horizontal thrust}

As can be seen in the information, its speed reduces from 1700 meters per second to 57 meters per second in about 600 seconds and that it is nearly right above the target area at the end of the target area. Obviously, the reduction of the horizontal speed is huge, and we assume that the thrust has been used to reduce the horizontal velocity all along in this process, and directs in the negative horizontal direction. Computation formula is as follows:

$$
m_{\min }=\frac{1}{3} * 2.4 * 10^{3}=0.8 * 10^{3} \mathrm{~kg} \quad a_{\max }=\frac{7500}{m_{\min }} \quad t_{\min }=\frac{1700-57}{a_{\max }} \quad h_{\min }=\frac{1}{2} * g * t_{\min }^{2}
$$

According to the above calculation, the solution of $h_{\min }$ is equal to $25077.7611 \mathrm{~m}$, which is far more than the actual vertical descent stage 12000 m,thus this model is wrong. So it also need to adjust the posture constantly to decline the increase of vertical speed in the process. The soft landing of a three-dimensional homogeneous sphere model is applicable to the main deceleration section, lander is far to the moon, and it is long to go through the distance, take the moon as a sphere, lander system adopts the way of constant thrust. It is used to solve the optimal control of dynamic movement process during the main reduction period.

\section{- Constant speed reverse thrust}

To consume least fuel can be converted to the shortest braking time, and thus assume that the thrust has always maintained maximum thrust $7500 \mathrm{~N}$. and the lander has been constantly adjusting posture in the main deceleration process, and making the thrust direction always keeps opposite with speed. According to the above algorithm, by using the method of computer simulation, we can get the drop height which is far more than $12000 \mathrm{~m}$ when the lander rate reaches $57 \mathrm{~m} / \mathrm{s}$. Therefore stage lander has not satisfied attitude adjustment movement during this process. Considering that the beginning lander vertical speed compared to horizontal speed is so small, it can be ignored, later with the increase of time, vertical velocity increases gradually, which need to adjust by adjusting its posture. Therefore consider lander do two phases of movement.

\section{- Constant speed reverse thrust after constant speed reverse resultant force}

According to the above analysis, the two-phases movements is established : the first phase of thrust is mainly used to reduce the lander's horizontal velocity, and the join force, consisting of the thrust and the gravity directs the negative horizon direction; The second stage thrust directs the negative velocity direction, and is mainly used to adjusting the appearance of the lander.

Based on the initial position and the landing height of the main reduction phase. The rate of transition point of the two movements is identified, which is $1000 \mathrm{~m} / \mathrm{s}$. Considering the error of calculation and the actual, when determining the second phase lander thrust direction, with the consideration of the motion state and state at the end of the speed change point, the conversion coefficient $\rho=1.38$ is added to the original measure speed direction.

\section{The solution based on the computer simulation method[5]}

From the above analysis, we only to find that only the last movement meets the fact.

Then the computer simulation method is employed to simulate the two-stage dynamic movements. Steps are as follows:

Step1: the first stage movement process: the step length is $0.1 \mathrm{~s}$, and the thrust is $7500 \mathrm{~N}$, with the joint force directing the negative horizontal direction, until the relative speed reduced to $1000 \mathrm{~m} / \mathrm{s}$. 
Step2: the second stage movement process: the lander fall from its relative speed being $1000 \mathrm{~m} / \mathrm{s}$ to above the surface $3 \mathrm{~km}$, and the time step is $0.1 \mathrm{~s}$. Every moment the direction of the thrust is the product of the last time negative speed Angle and 1.38, and the size is $7500 \mathrm{~N}$. Then, the dynamic movement of the stage is simulated.

According to the above steps, with the aid of Matlab computer simulation[6], each physical quantity calculation of the deceleration phase the results are as follows:

Run time: $\mathrm{t}=614.9 \mathrm{~s} \quad$ Landing height: $\mathrm{h}=12 \mathrm{~km} \quad$ Horizontal movement distance: $\mathrm{L}=534.83 \mathrm{~km}$

Above the surface $3 \mathrm{~km}$ height, the vertical speed $v_{\perp}=27.5395 \mathrm{~m} / \mathrm{s}$, the vertical speed horizontal velocity $\quad v_{/ /}==49.8271 \mathrm{~m} / \mathrm{s}$. For more intuitive lander movement situation, the time trajectory and the trajectory figure of the main the deceleration phase are drawn as follows:

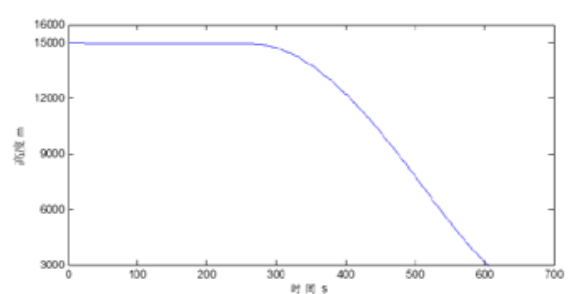

Fig4. The time trajectory figure

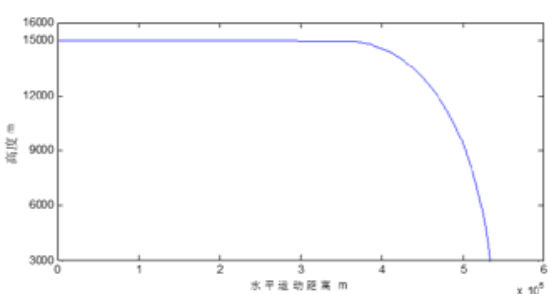

Fig5. The trajectory figure

As can be seen from the above two graphs, the lander first makes straight and level exercise, and then does parabolic movement. It is consistent to the fact that the initial speed is huge, lander almost do only horizontal, and also reflects the lander deceleration and well operation process according to the appearance of the navigation information movement. Analyzing the data, to complete this stage movement, the lander takes slightly more than $614.9 \mathrm{~s}$, level flight distance is $534.83 \mathrm{~km}$. Compared with the data collected, lander began to slow in front of the observation rainbow bay area about 500 $\mathrm{km}$, and took 600 seconds to reach $3000 \mathrm{~m}$ away from the surface. This shows our results' high accuracy.

\section{Summary}

The dynamic model based on the uniform sphere three-dimensional soft landing model, established to searching Chang'e-3's optimal trajectory in the main reduction process, appears working well. And the computer simulation method and the exhaustive method plays important roles in the calculation.

Compared with the searched video information, the accuracy of the results is verified.

\section{Reference}

[1] Xiao-chen Wang, sunny pan, five hin. Ten asked the goddess of the moon, reveal the goddess of the moon three spacecraft [J]. China aerospace commemorative edition, 2014, (1) : 15-26

[2] Pengji Wang. Optimized design research drops lunar soft landing trajectory and the guidance law [J]. Journal of aerospace, 2007, 28 (5) : 1175-1252

[3] The moon_baidu encyclopedia [EB/OL].http://baike.baidu.com/subview/1984/7833216.htm?Fr $=$

Aladdin.

[4] Wen-yan Zhou, Weilian Yang, Chang 'e 2 satellite orbit design [J]. Journal of spacecraft engineering, 2010, 19 (5) : 24 to 28

[5] ShouKui Si. Mathematical modeling algorithm and application [M]. Beijing: national defence industry press, 2014.

[6] Pengji Wang, He Zhang, Guang-ji Qu. The modeling and simulation of lunar soft landing flight dynamics and guidance control [J]. Chinese science E series, 2009, 39 (37) : 521-527 